\title{
LA MOTIVACIÓN Y RENDIMIENTO ACADÉMICO DE ESTUDIANTES EN LA IES NUESTRA SEÑORA DE ALTA GRACIA
}

\author{
Julio YANA MOLLEAPAZA ${ }^{1}$
}

\begin{tabular}{|lcc|}
\hline Recibido & $:$ & 30.04 .2020 \\
Aceptado & $:$ & 29.06 .2020 \\
Publicado & $:$ & 06.07 .2020 \\
\hline
\end{tabular}

RESUMEN: La investigación surge de la necesidad de establecer una relación entre la motivación y el rendimiento académico de estudiantes en la institución educativa secundaria Nuestra Señora de Alta Gracia de la ciudad de Ayaviri, teniendo primeramente la observación directa, aplicando la encuesta y luego la entrevista. El objetivo de trabajo es determinar la relación que existe entre la motivación y rendimiento académico en función de dos variables: la motivación y rendimiento académico. El tipo de investigación es descriptivo y correlacional. La muestra está conformada con 270 estudiantes, para la recolección de datos se realizaron encuestas y entrevistas para caracterizar la motivación con las siguientes dimensiones: inteligencia emocional, autoestima, y su motivación en la institución educativa y así la convivencia democrática, se establece los resultados en las Áreas de: Comunicación, Matemática, Ciencias Sociales y Ciencia y Ambiente. Se concluye si existe una correlación positiva moderada el valor de chi cuadrada calculada es de 15.08, realizando una tabulación para explicar la investigación.

Palabras claves: Motivación, Rendimiento Académico.

\section{Motivation and academic performance of students at the IES Nuestra Señora de Alta Gracia}

\begin{abstract}
The research arises from the need to establish a relationship between motivation and academic performance of students at the Nuestra Señora de Alta Gracia secondary educational institution in the city of Ayaviri, having First, direct observation by applying the survey and then the interview as an objective, I plan to determine the relationship between motivation and academic performance. In addition, the problem is described, analyzed and explained in terms of two variables: motivation and academic performance, whose hypothesis that he proposed is the degree of motivation and academic performance. Research type is descriptive and correlational. The sample is made up of 270 students, for data collection, there were conducted surveys and interviews to characterize motivation with the following dimensions: emotional intelligence, self-esteem, and their motivation in the educational institution and thus democratic coexistence, the results are established. in the areas of: Communication, Mathematics, Social Sciences and Science and Environment. It is concluded if there is a moderate positive correlation, the calculated chi square value is 15.08 , making a tabulation to explain the research.
\end{abstract}

Keywords: Motivation, Academic Performance.

\footnotetext{
${ }^{1}$ Universidad Nacional del Altiplano de Puno. Email: julioyana2014@gmail.com
} 


\section{Journal of the Academy $|76|$}

\section{INTRODUCCIÓN}

La preocupación e incertidumbre en la actualidad y como docente de aula, investigue como lograr que los estudiantes de educación secundaria adquieren aprendizajes significativos para un buen rendimiento académico, en particular en la Institución Educativa que vengo laborando lo realizo esta seria investigación, identificando que muchos factores dificultan el éxito del proceso de enseñanza aprendizaje, para evitar el detrimento de la mejora de la calidad educativa. En conformidad con uno de los objetivos estratégicos que contempla el Proyecto Educativo Nacional al 2021. (MINEDU, Proyecto Educativa Nacional al 2021. La Educación que queremos para el Perú, 2006) "Estudiantes e instituciones que logran aprendizajes pertinentes y de calidad". La campaña por las mejoras de los aprendizajes, el 16 de diciembre de 2011 se publicó en el diario oficial:

"El Peruano"; en el numeral cinco presenta la Movilización Nacional por la Transformación de la Educación "Cambiemos la Educación, Cambiemos Todos", "Campaña por la mejora de los Aprendizajes y la Escuela que Queremos", "Todos aprendemos nadie se queda atrás" y "Un buen maestro cambia tu vida", "La nota más alta".

Lo que traduce en que los docentes debemos de motivar y estar bien preparados para enseñar y dirigir a los estudiantes, "motivarlos", maestros bien preparados ejercen profesionalmente la docencia" según (MINEDU, Manual del Buen Desempeño Docente, 2015). Los profesionales de la educación tienen la idea o la motivación que es el elemento clave para el aprendizaje óptimo de los estudiantes de una o más áreas curriculares, por eso se entiende que los factores afectivos dentro de los cuales se encuentra la motivación, no pueden aparecer desvinculados de los factores cognitivos que son los que tradicionalmente se han visto privilegiados en las aulas, la Institución Educativa cuenta con su Proyecto Educativo Institucional, se concluye con cuya visión compartida:

"Al 2021 somos una IES líder con alto nivel académico y de gran prestigio; destacando en ciencia, cultura y deporte, que garantiza la formación holística y axiológica de los estudiantes en un contexto pro activo e innovador, con una infraestructura moderna ecológica en armonía con el medio ambiente y la sociedad".

En el proceso de la investigación, se trata de identificar las dimensiones e indicadores de motivación y rendimiento académico que la educación como uno de los pilares de la sociedad, es pues la columna vertebral para un desarrollo de un país sostenible y actualmente la investigación sobre este tema motivacional, se vienen insertando en las instituciones educativas, (MINEDU, Diseño Curricular Nacional, Rutas de Aprendizaje y Mapas de Progreso., 2015) más aun con las nuevas propuestas del Ministerio de Educación, muchos maestros se preguntan cómo puedo motivar a mis alumnos en clase, si ellos se distraen con sus dispositivos móviles o celulares, en este contexto surge una cuestión muy significativa en los escolares, al docente le parece un poco difícil de acentuar el proceso de motivación escolar, muchas investigaciones se han realizado sobre todo en los países extranjeros, caso peruano es reciente este término utilizado, muchos educadores afirmaban que la motivación es hacer querer al alumno, hacerle gustar, que se animen, que tomen atención, que nos escuche sin conversar, mirar sin mover, estar firmes, etc. La reflexión nos llevó que se trata de falta de buena motivación para el buen rendimiento académico en los estudiantes, por eso se considera la motivación es uno de los factores psicológicos y emocionales, que juega un papel muy significativo en el proceso de enseñanza aprendizaje.

\section{DESARROLLO}

\section{Bases Teóricas}

Para entender y comprender la motivación es como una atracción que va impresionar hacia una acción al estudiante y permite aceptar el esfuerzo para conseguir ese logro tiene la importancia de estudiar con la sana intención de lo que es la motivación, además que tienen el gran potencial de elevar el nivel de su rendimiento académico, dicho estudio lo realizo porque existe la necesidad urgente de conocer 


\section{Journal of the Academy $|77|$}

cuan motivados están los estudiantes en la institución educativa en relación a su rendimiento académico. La investigación sirve para seguir contribuyendo y sea muy beneficioso en el plan de mejoras motivando a los y las estudiantes hacia el buen rendimiento académico óptimo y significativo en la institución, de hecho que va a generar intercambio de experiencias de reflexión, propuestas de innovación en las aulas, propiciando el buen clima escolar, el cambio de actitudes de los docentes y estudiantes con la aplicación de nuevas estrategias metodológicas actuales, maestros comprometidos con la educación, asimismo el estudiante para que nunca más sea desmotivado en el aula y en el contexto mundial de competitividad y practica de valores que aún está en riesgo, podemos construirla solo siendo motivados y optimismo buscando dialogo y comunicación.

Según (Dornely, 2005, pág. 79) "La motivación es un concepto abstracto, un concepto hipotético que existe para poder explicar la razón por la cual la gente se comporta y piensa de la forma que lo hace (...) la motivación está relacionada con uno de los aspectos más básicos de la mente humana y esta tiene un rol determinante en el éxito o fracaso en cualquier situación de aprendizaje".

Actualmente se considera que el proceso de la motivación es desarrollado por todo un conjunto de actores de un determinado proceso, y que la función del profesor, facilitador o motivador es ayudar a que cada estudiante que alinee y ordene su aprendizaje, puesto que la motivación no consiste en imponer una voluntad sobre otra, más bien de convencer a todas las voluntades para lograr un objetivo común. Sin embargo, para poder saber si esta motivación influye en el rendimiento académico de los estudiantes; para ello es necesario establecer el tipo de motivación escolar que se observa con mayor detalle que la motivación escolar es muy fundamental en la práctica docente así como plantea el Ministerio de Educación sobre el concurso de las Buenas Prácticas Docentes anualmente, en cuanto a valores y convivencia democrática la estrategia de normas o reglas de juego en sus estudiantes, en todo caso resulta de un concepto de necesidades o intereses de carácter biológico, psicológico y social. se evidencian estudiantes desmotivados o con un reducido baja autoestima, se aburren fácilmente no toman iniciativa e interés por el aprendizaje y otros con bajas calificaciones en sus notas trimestrales, la repitencia, deserción escolar o retirado, traslados externos, inasistencia a clases, distraídos con celulares en las aulas, en el patio o espacios deportivos, conversando entre ellos otros practicando deportes, asimismo se han verificado también en las cabinas de internet o videojuegos pasando el día horas y horas, ante esta situación se debe a diversos factores como ya dijimos anteriormente.

(Barriga, 2010) La más vista a través de un vídeo sobre motivación me refiero a (Atkinson, 1958) sostiene a la motivación como la activación de una tendencia a actuar para producir uno o más efectos, se resume que busca demostrar que la persona influye la necesidad de logro que posee. Para (A., 1990), el propone una escala en las necesidades que divide cinco grupos o niveles como básicas, la supervivencia, sociales, crecimiento, autoestima, autorrealización y trascendencia, para el autor la satisfacción de las necesidades básicas se siente motivado para alcanzar a cubrir las necesidades de crecimiento y autoestima hasta convertirse en un sujeto auto realizado. Continuando en el mismo video me pareció muy interesante de (Young, 1961), define a la motivación como el proceso para despertar la acción, sostener la actividad en progreso y regular el patrón de actividad y para el señor. (Rodriguez, 2009).

"Señala en su objetivo concretamente las diferencias de género en motivación, estrategias y rendimiento académico en dicho nivel educativo, en el segundo lugar en el intento de profundizar en esta relación entre motivación, estrategia y rendimiento, se llevan a cabo una serie de análisis de regresión para observar la incidencia de las metas académicas sobre el empleo de estrategias de estudio y sobre el rendimiento académico de los estudiantes".

(Bueno, 1993) "La motivación en los alumnos de bajo rendimiento académico": desarrollo y programa de intervención el objetivo es llevar a cabo un estudio evolutivo transversal en una población escolarizada sobre algunos aspectos motivacionales a luz de una teoría y sobre esos resultados, diseñar y verificar la eficacia de un programa de enriquecimiento motivacional, como objetivo general es determinar el grado de relación que existe entre la motivación y rendimiento académico de los estudiantes de la institución educativa y específicos es identificar los tipos y niveles de motivación en 


\section{Journal of the Academy $|78|$}

los estudiantes, identificar el rendimiento académico de los estudiantes en las áreas curriculares de: Comunicación, Matemática, Ciencias Sociales, Ciencia y Ambiente y correlacionar la relación que tienen las dimensiones e indicadores de la motivación y los resultados según su rendimiento académico de los estudiantes.

Recientemente desde la década de los años setenta es decir del siglo pasado se empezó a investigar en nuestro país sobre la variable motivación, esto lo señala (Remon, 2013), son pocas las investigaciones que se han realizado peor aún sobre la motivación escolar, indago sobre las relaciones entre la motivación, estrategias de aprendizaje, auto concepto y rendimiento académico en escolares de quinto de Secundaria y se encontró que las variables más relacionados al rendimiento son el auto concepto académico y los componentes motivacionales:

Lo señala nuevamente con (Remon, 2013, pág. 123). "El clima social familiar y motivación académica en estudiantes de tercer y cuarto grado de secundaria pertenecientes a los colegios católicos de Lima Metropolitana, el sustento basado en una motivación interna para el aprendizaje lo externo para castigo o recompensa, a esto se puede añadir mitos o creencias como a mí me gusta, a mí no me gusta estudiar o realizar las tareas, también se puede predecir que los escolares en esos grados de estudios son la columna vertebral".

Por una parte, (Yactayo, 2010), en sus conclusiones menciona:

Se confirmó la hipótesis general que suponía que, si existe una relación entre la motivación de logro académico y rendimiento académico, esta relación es positiva pero moderada, también señala diciendo se estableció una relación significativa entre el componente acciones orientadas al logro y rendimiento académico, se refiere a la relación moderada, finalmente señala que existe una mayor cantidad de mujeres que varones con motivación de logro académico alta.

En este contexto se puede deducir que el alumno se ven entre dos necesidades concretas una la de alcanzar el éxito en el aprendizaje y la otra la de evitar el fracaso en el aprendizaje, el autor muestra que el éxito siempre perdura en una relación del aprendizaje con su rendimiento.

\section{Materiales y metodología}

Materiales como fuentes bibliográficas como libros, trabajos de investigaciones. revistas, artículos científicos, web, encuestas dirigidas y realizables, entrevistas directas, videos, fotografías, etc, dentro de la institución educativa y en el ámbito local, los métodos como inductivo, deductivo, análisis, científico y acción. Observación directa como la escala de Motivación y fichas adaptadas del investigador, así como test, encuestas y análisis psicométrico, en cuanto a instrumentos como: registros de evaluación, lista de cotejos, agendas del alumno.

Como la variable independiente la motivación como indicadores, inteligencia emocional, autoestima personal, cuán motivado me siento en la institución educativa y en general y la convivencia democrática. Las técnicas e instrumentos que se utilizó el cuestionario está conformado de veinte ítems divididos en cuatro dimensiones como: inteligencia emocional, autoestima personal, cuan motivado me siento en la institución y en general con tres escalas nunca, a veces y siempre, también se identificó las notas del primer trimestre académico de evaluación de las áreas curriculares ya mencionadas, para determinar el grado de relación que existe entre la motivación y rendimiento académico de los estudiantes. Para la prueba de hipótesis, Ha: Existe la relación entre la motivación y el rendimiento académico significativa en los estudiantes Ho: No existe la relación entre la motivación y el rendimiento académico en los estudiantes de la institución secundaria Nuestra Señora de la ciudad Ayaviri. 
Journal of the Academy $|79|$

\begin{tabular}{|c|c|c|c|c|}
\hline \multirow{3}{*}{ INDICADORES } & \multicolumn{4}{|c|}{ MOTIVACIÓN ESCOLAR } \\
\hline & SIEMPRE & A VECES & NUNCA & TOTAL \\
\hline & $\mathbf{N}$ & $\mathbf{N}$ & $\mathbf{N}$ & $\mathbf{N}$ \\
\hline LOGRO DESTACADO & 2 & 3 & 0 & 5 \\
\hline RENDIMIENTO LOGRO PREVISTO & 45 & 41 & 8 & 94 \\
\hline $\begin{array}{l}\text { EN PROCESO } \\
\text { ACADÉMICO }\end{array}$ & 44 & 105 & 20 & 169 \\
\hline EN INICIO & 0 & 2 & 0 & 2 \\
\hline TOTAL & 91 & 151 & 28 & 270 \\
\hline
\end{tabular}

Fuente: Prueba de hipótesis.

\section{Resultados y discusiones}

¿Cuyo propósito de la investigación fundamentalmente está orientado a identificar y correlacionar entre la motivación del educando y su rendimiento académico para un aprendizaje optimo y significativo, dicho sea de paso, cuanto más motivado los alumnos y alumnas sean, mejor será su rendimiento académico, es decir el alumno cuanto más hace preguntas más aprenderá?, es cierto cuantas más preguntas aprendes más, quien la sigue la consigue, quien estudia triunfa, etc. Al respecto definimos que de la motivación escolar es la fuerza como la gran energía que se tiene para optimizar el logro en el aprendizaje, según (Barriga, 2010) "a los alumnos ahora ya no les interesa la escuela", "mis estudiantes no llegan motivados para trabajar en clase", "solo les gusta el relajo, vagancia", etc., según nuestra prospectiva aquí el docente es la persona que no solo orienta, facilita el aprendizaje, más bien promotor de la motivación, esa es su vocación, de que se dice maestros de vocación o de ocasión, por su parte (Fischman, 2000, pág. 146) señala la mejor profesora del mundo es la señora Thompson.

De acuerdo al nuevo Currículo Nacional de Educación Básica, que está en vigencia en los colegios de la Jornada Escolar Completa, el caso la localidad de Ayaviri, la inserción de las dos horas de tutoría en los colegios, va inducir habrá más acercamiento al estudiante en su formación integral ya que el docente es promotor de la motivación y beneficioso para los estudiantes. La falta de motivación es un gran problema también en el colegio, según (Fischman, 2000, págs. 16, 17), afirma lo siguiente: cerca del cuarenta por ciento de estudiantes de secundaria manifiesta sentirse totalmente desmotivado para estudiar, estudiantes aburridos, poco atentos y se esfuerzan poco. La autoestima tiene varias definiciones como acto valorativo de nuestra manera de ser, nuestras actitudes, otra casa es decir y otra cosa es hacer, la mente es la mejor arma, lo que piensas, lo sientes, lo amas, lo odias, etc., la estudiante saco de su mochila su libro de Telmo Salinas de Manual de Psicología, finalmente termino, profesor dijo usted si está preparado, gracias. Si bien es cierto la autoestima nace cuando crees, es decir cuando te amas a ti mismo de verdad, sin mentiras, es decir una persona tiene mente positiva, sea optimista, la realidad, la verdad es el amor señores miembros del jurado del premio, tanta situación, muchas veces se habla de autoestima, pero en realidad es observar a profundidad la realidad en el tiempo y en espacio.

Se sufre y se lucha en la vida, controlando nuestras emociones y sentimientos, muchas personas se acaban sus vidas, por parejas disfuncionales, el vicio, las frustraciones, la ira, odio. (Goleman, 2010, pág. 125), menciona: "A lo largo de la vida, las dos emociones más inútiles son la culpabilidad por lo que se ha hecho y la otra preocupación por lo que se podría hacer, son grandes despilfarros..." (Goleman, 2010, pág. 127), sostiene: "el control de emociones puede utilizar esa ansiedad anticipatoria, por ejemplo, sobre un examen o una charla próxima, para motivarse a sí mismo, prepararse adecuadamente y en consecuencia hacerlo bien, rendimiento escolar, muchos alumnos no culminan sus estudios de educación secundaria",

Si bien algunos dejan sus estudios por problemas académicos de las instituciones educativas de Ayaviri, el caso de siete estudiantes, la mayoría piensa que pudo haber dificultades, no obstante es el resultado o logro de todas las capacidades, habilidades y destrezas que pone empeño e interés el alumno adquiriendo las nuevas formas de aprendizajes, ahí juega un papel muy importante de la autoeducación, sirve para toda la vida, de ahí que un filósofo Víctor Afanasiev afirmaba la educación es el alimento del hombre. 


\section{Journal of the Academy $|80|$}

\section{CONCLUSIONES}

La motivación y rendimiento académico al tabular los resultados se plantea si existe una correlación positiva moderada el valor de chi cuadrada calculada es $X_{c}^{2} 15.08$ es mayor a la variable chi cuadrada tabulada $X_{t}^{2}=12.59$, entonces se rechaza la hipótesis nula y se acepta la hipótesis alterna según el cuadro del anexo adjunto, en resumen lo que quiere decir que si existe relación directa y significativa entre la motivación y rendimiento académico en los estudiantes de la institución educativa Nuestra Señora de Alta Gracia de la ciudad de Ayaviri.

Un buen porcentaje de los escolares en el ítems dos indican el $75 \%$ a veces llegar a dudar de sus sentimientos, de acuerdo a nuestra investigación los adolescentes que adolecen tienen toda la razón, en ella se acentúa la motivación y es natural en los estudiantes de secundaria porque existe esa razón, de ahí que la inteligencia emocional es sumamente como el control de los impulsos, seguido por los sentimientos, ese tema es transversal en los colegios, de hecho refleja la orientación hacia los estudiantes. El 68\% se manifiesta que si tengo que decir no lo dicen, la privacidad, la baja autoestima personal, la desconfianza, el estrés adolescente, utilización de los aparatos dispositivos el celular y la ansiedad son fuertes tensiones, de ahí que se evidencia en que se distraen fácilmente o a veces no quieren decir, resulta como cada individuo es lo que es, a cada paso encontramos una situación difícil de entender a los escolares, cada estudiante es un problema, el 3\% nunca afrontan o se enfrentan a nuevos retos el entusiasmo es un estado de consciencia que refleja en la condición de uno mismo es triunfador o derrotado.

Cuan motivados se sienten, el $78 \%$ afirman que los docentes a veces son aburridos, coléricos, autoritarios, personalistas esto significa que la investigación evidencia el promotor de la motivación es el maestro de escuela como, el estudiante llega con bastante atención a las aulas, de ver esa actitud de sus docentes cambian así como dice el niño o púber es el reflejo de su profesor y respecto a la convivencia democrática el 57\% participan en forma disciplinada, democrática respetando a los demás compañeros, cierto; nuestros alumnos son la razón de ser, por ende de la educación, debo de indicar en el colegio se venía trabajando con el Programa de Auditores Juveniles Escolares de la Contraloría General de la República por tercera vez, algo cambiaría en la transparencia, el trato a los escolares, la tolerancia y responsabilidad, algo ha mejorado.

En el área de matemática en su rendimiento académico con el $66 \%$ está en proceso, el área la más cuestionada y mucha prioridad se da en la institución educativa, resulta esta conclusión tal vez, la motivación está pésimamente, sin embargo, el rendimiento es bajo o en proceso. En Comunicación con el 58\% en el rubro en proceso se ubica la mayoría de porcentaje, según los registros de evaluación del primer trimestre, también el área menos cuestionada por los padres de familia y también prioridad de la comunidad educativa, esto resulta que la motivación es muy fundamental. Leer para vivir, comprensión lectora, asimismo proyectos de lectura, es preocupante esta situación sobre el rendimiento académico, en el área de Ciencias Sociales está en logro previsto con un 51\% de ponderación, significa los docentes motivan son sociables, están en la mejora de aprendizajes, nos orgullece ver este resultado, de ahí que la historia hacen los hombres, además los estudiantes comprenden el espacio y tiempo histórico, la identidad cultural, se ha visto que existe mayor de docentes motivadores que siempre están pendientes de sus estudiantes ejemplo en la formación en la entonación del Himno Nacional en los días de formación.

Más del 63\% está en proceso, en Ciencia y Ambiente, es parte muy importante en la investigación, preocupa a la comunidad educativa de detallar estos resultados que nada son fructíferas, la indagación, la experimentación debe estar al alcance de los estudiantes, sin embargo a nuestro criterio, la motivación es muy importante en las áreas curriculares, en la once áreas, sin embargo hay preocupación aún todavía enfrentar a estudiantes que están con la tecnología el usos de celulares, ya saben la información pero las competencias hay que desarrollarlas motivando y despertando su interés por aprender. 


\section{REFERENCIAS BIBLIOGRÁFICAS}

A., M. (1990). Escala de necesidades. En Motivación como necesidad (pág. 245).

Barriga, F. y. (2010). Estrategias docentes para un aprendizaje significativo: Una interpretación constructivista. Punta Santa Fe - México: 3ra Edición.

Bueno, J. A. (1993). La motivación en los alumnos de bajo rendimiento académico. Madrid - España: Impresiones Lda.

Dornely. (2005). Teorías de la motivación y problemas del Aprendizaje en los alumnos. Barcelona: Impress. 63, P. E. (s.f.).

A., M. (1990). Escala de necesidades. En Motivación como necesidad (pág. 245).

Barriga, F. y. (2010). Estrategias docentes para un aprendizaje significativo: Una interpretación constructivista. Punta Santa Fe - México: 3ra Edición.

Bueno, J. A. (1993). La motivación en los alumnos de bajo rendimiento académico. Madrid - España: Impresiones Lda.

Dornely. (2005). Teorías de la motivación y problemas del Aprendizaje en los alumnos. Barcelona: Impress.

Fischman, D. (2000). Motivación 360. 4ta Edición.

Goleman, D. (2010). La inteligencia Emocional porque es más importante que el consciente intelectual. Buenos Aires: Ediciones Bestseller Mundial.

MINEDU. (2006). Proyecto Educativa Nacional al 2021. La Educación que queremos para el Perú. Lima -Perú: Consejo Nacional de Educación.

MINEDU. (2015). Diseño Curricular Nacional, Rutas de Aprendizaje y Mapas de Progreso. Lima Perú: Ministerio de Educación.

MINEDU. (2015). Manual del Buen Desempeño Docente. Lima - Perú.

Remón, S. (2013). Relaciones entre la motivación, estrategias de aprendizaje, autoconcepto y rendimiento académico en escolares de $5^{\circ}$ de secundaria. Lima - Perú: Facultad de Psicología.

Rodríguez, G. (2009). Motivación, Estrategias de Aprendizaje y rendimiento académico en estudiantes de E.S.O. Coruña: 1ra Edición.

Yactayo, Y. L. (2010). La motivación y Logro académico y rendimiento en alumnos de secundaria de la Institución Educativa del Callao Universidad San Ignacio de Loyola. Lima- Perú: Facultad de Educ. 1ra Edición.

Young. (1961). vídeo. 